\title{
Landtagswahlen in Niedersachsen 1970 bis 2003: Landespolitik als wichtiger Einflussfaktor
}

\author{
Evelyn Bytzek und Susumu Shikano
}

Landtagswahlen stellen die Wähler vor eine komplexere Entscheidungssituation als beispielsweise Bundestagswahlen, da bei ihnen zwei unterschiedliche Bewertungsgrundlagen nämlich landes- und bundespolitische Faktoren - für ihr Verhalten eine Rolle spielen können. Im Folgenden wird anhand der Wahlen zum Niedersächsischen Landtag untersucht, welche der beiden Bewertungsgrundlagen bei welcher Wahl in den Vordergrund trat.

\section{Historische Entwicklung der niedersächsischen Landtagswablen}

1947 fand die erste Landtagswahl im nach dem Zweiten Weltkrieg neu gebildeten Bundesland Niedersachsen statt (vgl. für Wahlergebnisse Abbildung 1). Das damalige Wahlsystem wich vom heutigen Bundeswahlrecht in zwei wesentlichen Punkten ab: Erstens wurde mit einer Einzelstimme gleichzeitig ein Kandidat im Einerwahlkreis und dessen Partei auf Landesebene gewählt. Zweitens war keine Sperre gegen den Einzug von Splitterparteien vorgesehen. ${ }^{1}$ Die erste Wahl führte mit sechs im Landtag vertretenen Parteien (SPD, CDU, $\mathrm{NLP}^{2}$, FDP, KPD, Zentrum) zu einer überschaubaren Lage. Dies änderte sich mit der Landtagswahl 1951, durch die neun Parteien in den Landtag gewählt wurden, darunter die rechtsradikale SRP (Sozialistische Reichspartei), die ihre Sitze jedoch nach dem Verbot durch das Bundesverfassungsgericht 1952 aberkannt bekam.

Wie auch auf Bundesebene trat in der Folgezeit eine starke Konzentration des Parteiensystems in Niedersachsen ein. So sank die Zahl der Fraktionen im Landtag schon bei der Landtagswahl 1955 auf acht und reduzierte sich 1959 nach der Einführung einer Sperrklausel von fünf Prozent auf fünf. Dieser Prozess wurde 1963 abgeschlossen, als nur noch die auch im Bundestag vertretenen Parteien SPD, CDU und FDP Mandate errangen. Mit der Wahl 1967 konnte jedoch auch die NPD in den Landtag einziehen. Dies war keine dauerhafte Erscheinung. Die Wahl 1970 brachte durch den Wegfall der FDP, die mit 4,4 Prozent an der Sperrklausel scheiterte, eine weitere Konzentration des parlamentarischen Parteiensystems mit sich. Nachdem 1974 mit der FDP wiederum drei Parteien im Landtag vertreten waren, wiederholte sich dieser Fall 1978, als die Freidemokraten nur 4,2 Prozent der Stimmen bekamen. Auch die Grünen traten 1978 an, konnten jedoch nur 3,9 Prozent erzielen. Dies änderte sich 1982, als sie mit 6,5 Prozent der Stimmen erstmals in den Niedersächsischen Landtag einzogen. Seitdem stellen sie im Gegensatz zur FDP eine beständige Größe im parlamentarischen Parteiensystem Niedersachsens dar. Bei der Landtagswahl 1990 wurde erstmals ein am bundespolitischen Vorbild orientiertes Zweistimmensystem

1 Es ist zu beachten, dass dieses Wahlsystem nicht weit von dem bei der Bundestagswahl 1949 angewandten Wahlrecht entfernt ist. Auch bei der ersten Bundestagswahl wurden ein Kandidat und dessen Partei mit einer Einzelstimme gewählt, wobei die Fünf-Prozent-Sperrklausel nicht auf Bundesebene, sondern nur auf Landesebene galt.

2 Niedersächsische Landespartei. Diese Partei nannte sich im selben Jahr der ersten Landtagswahl in Deutsche Partei (DP) um. 
angewandt. Obwohl die FDP diese Wahlsystemänderung mit der Hoffnung auf so genannte Leihstimmen durchsetzte ${ }^{3}$, schaffte sie sowohl 1994 als auch 1998 den Einzug in das Landesparlament nicht. ${ }^{4}$ In den 1960er Jahren kristallisierte sich also ein Drei- bis Vierparteiensystem heraus, das dem auf Bundesebene sehr stark glich. Dies kann als erster Hinweis auf einen bundespolitischen Einfluss dienen.

Einen weiteren Fingerzeig erhält man beim Blick auf die niedersächsischen Landesregierungen. Diese sind von einer wechselnden Dominanz der beiden großen Parteien geprägt. Von 1947 bis zum Ende der sechziger Jahre stellte die SPD mit einer Unterbrechung von 1955 bis 1957 (konservative Regierung unter Führung Heinrich Hellweges von der DP) ständig den Ministerpräsidenten (mit Hinrich Wilhelm Kopf $f^{5}$, der nach seinem Tod 1961 von Georg Diederichs abgelöst wurde, der das Amt 1970 wiederum an Alfred Kubel weitergab). Die schwachen Ergebnisse der CDU in der Anfangsphase liegen vor allem darin begründet, dass das konservative Lager in Niedersachsen durch die Existenz der Deutschen Partei (DP), des Bundes der Heimatvertriebenen und Entrechteten (BHE), des Zentrums und weiterer rechtsextremer Parteien stark fragmentiert war. Zwar war auch die KPD bis 1956 mit 2 Sitzen im Niedersächsischen Landtag vertreten; dennoch kann damit von einer Fragmentierung des linken Lagers keine Rede sein, was die Dominanz der SPD begünstigte. Erst 1974 stellte die CDU durch eine außergewöhnlich hohe Wählermobilisierung zum ersten Mal die größte Fraktion im Landtag. ${ }^{6}$ Doch zunächst regierte die SPD unter Alfred Kubel in Koalition mit der FDP weiter. Dies änderte sich 1976 durch den Rücktritt Kubels und der unerwarteten Präsentation eines aussichtsreichen Gegenkandidaten, Ernst Albrecht, durch die CDU.7 Danach blieb dieser bis zur Landtagswahl 1990 Ministerpräsident, die CDU stärkste Partei. Diese Rolle fiel seit dem überraschenden Sieg Gerhard Schröders 1990 der SPD zu. Bis 2003 stellte sie die stärkste Fraktion und konnte nach einer ersten Phase von 1990 bis 1994, in der sie zusammen mit den Grünen die Regierung bildete, zwei

3 Vgl. Reinhold Roth, Die niedersächsische Landtagswahl vom 13. Mai 1990, in: ZParl, 21. Jg. (1990), H. 3, S. 449 - 460. Eine Leihstimme wird von einem Anhänger einer größeren Partei für den kleineren Koalitionspartner vergeben, damit der Einzug der kleineren Partei und damit die von der größeren Partei geführte Koalitionsregierung ermöglicht werden. Vgl. Susumu Shikano I Michael Herrmann / Paul W. Thurner, Explaining German strategic list voting with a two-step random utility approach, Paper vorgetragen beim Annual Meeting der Midwest Political Science Association, 2006.

4 Vgl. Uwe Thaysen, Parlamentarismus in Niedersachsen: Der Landtag im Leineschloss, in: Siegfried Mielke / Werner Reutter (Hrsg.), Länderparlamentarismus in Deutschland, Wiesbaden 2004, S. $277-306$.

5 Für eine detaillierte Darstellung der Legislaturperiode 1955 bis 1959, die zunächst die Bildung einer bürgerlichen Koalition sah, die 1957 jedoch von einer Großen Koalition unter Beteiligung der DP abgelöst wurde, vgl. Helmut Beyer / Klaus Müller, Der Niedersächsische Landtag in den fünfziger Jahren. Voraussetzungen, Ablauf, Ergebnisse und Folgen der Landtagswahl 1955, Düsseldorf 1988.

6 Vgl. Klaus Wettig, Die niedersächsische Landtagswahl vom 9. Juni 1974. Entscheidung der mittelständischen „Partei der Nichtwähler“ zugunsten der CDU?, in: ZParl, 6. Jg. (1975), H. 4, S. $404-419$.

7 Vgl. Wolfgang Jüttner / Klaus Wettig, Die niedersächsische Landtagswahl am 4. Juni 1978: Wem schadeten die „Grünen“, wem die Nichtwähler?, in: ZParl, 10. Jg. (1979), H. 1, S. 17 - 34. Für eine detaillierte Darstellung des Regierungswechsels 1976: Werner Pöls, Regierungswechsel in Hannover. Die Ereignisse im Niedersächsischen Landtag vom 14. Januar bis 6. Februar 1976, Hannover 1977. 
Wahlperioden lang allein regieren. 2003 siegte jedoch die CDU vor allem dank ihres Spitzenkandidaten Christian Wulff.

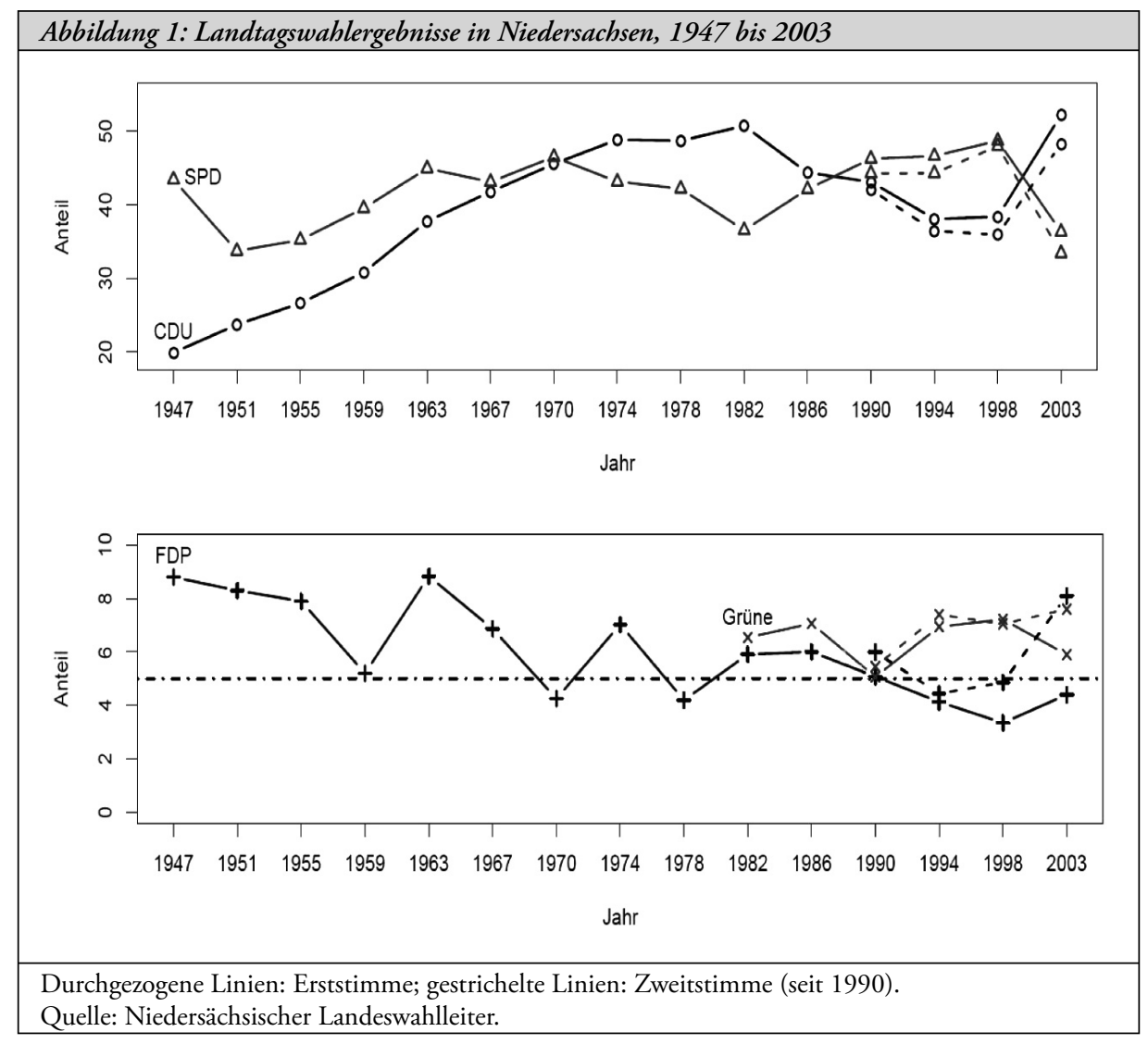

Betrachtet man dieses Wechselspiel der großen Parteien, findet man eine gewisse spiegelbildliche Entwicklung auf der bundespolitischen Ebene. In der Anfangsphase der Bundesrepublik unter der Kanzlerschaft der CDU (1949 bis 1969) dominierte die SPD in Niedersachsen (1947 bis 1974), ebenso wie in der zweiten Hälfte der Kohl-Ära (1990 bis 2003). Dagegen kam zur Zeit der sozial-liberalen Bundesregierungen (1969 bis 1982) und unter der Kanzlerschaft von Gerhard Schröder (1998 bis 2005) die CDU an die Landesregierung (1976 bis 1990 und seit 2003). Dies könnte ein Zeichen dafür sein, dass die Landtagswahlen von der Bundespolitik in Form von Protest-Wahlen beeinflusst wurden oder von Überlegungen, Politik durch „divided government" auszubalancieren. ${ }^{8}$ Der Wechsel der Wählerpräferenzen von den Regierungs- auf die Oppositionsparteien im Bundestag auf der Landesebene erfolgt jedoch mit einer gewissen Verzögerung. Dies könnte ein Hinweis darauf sein, dass die Unzufriedenheit mit der Bundesregierung eine wesentliche Rolle bei

8 Vgl. hierzu Alberto Alesina / Howard Rosenthal, Partisan Politics, Divided Government, and the Economy, Cambridge 1995. 
der Wahlentscheidung zu Wechselzeitpunkten gespielt hat. Dieser Annahme steht gegenüber, dass die personelle Kontinuität bei den Ministerpräsidenten Niedersachsens sehr hoch ist und Regierungswechsel häufig mit Personalveränderungen in der alten Mehrheitspartei einhergingen. So erfolgte die Regierungsübernahme durch die CDU 1976 nach dem Rücktritt Kubels. 1990 trat der amtierende Ministerpräsident Ernst Albrecht zwar noch einmal als Spitzenkandidat an, präsentierte jedoch schon vor der Wahl seine zukünftige Nachfolgerin Rita Süssmuth. 2003 folgte Sigmar Gabriel auf Schröder als Spitzenkandidat der SPD. ${ }^{9}$ Hieraus ließe sich der Einfluss landespolitischer Faktoren auf das Wahlverhalten ableiten.

Aus der Betrachtung der Wahlergebnisse und Regierungen in Niedersachsen geht also nicht deutlich hervor, ob bundes- oder landespolitische Aspekte das Verhalten bei Landtagswahlen mehr prägen. Es stehen sich die Zufriedenheit mit der Bundesregierung und die Attraktivität der Spitzenkandidaten als mögliche Einflussgrößen gegenüber, was im Folgenden anhand von Umfragedaten erörtert wird.

\section{Zufriedenheit mit der Bundesregierung und Attraktivität von Landespolitikern in Niedersachsen}

Zur Untersuchung landes- und bundespolitischer Einflüsse werden Umfragedaten zu acht niedersächsischen Landtagswahlen zwischen 1970 und 2003 herangezogen. ${ }^{10}$

Die Bewertung der Spitzenkandidaten als Landesfaktor unter Berücksichtigung der Zufriedenheit mit der Bundesregierung als Bundesfaktor ergibt Folgendes (vgl. Tabelle 1): 1970 konnte die SPD ein letztes Mal die meisten Stimmen erringen, bevor 1974 erstmals die CDU stärkste Partei im Niedersächsischen Landtag wurde und bis zur Wahl 1990 blieb. Da sich die Beliebtheit der beiden Spitzenkandidaten, Wilfried Hasselmann für die CDU und Alfred Kubel für die SPD, jedoch kaum unterschied (auch statistisch), könnte für den Wahlsieg 1970 die sehr große Zufriedenheit mit der sozial-liberalen Bundesregierung verantwortlich sein. Dies deutet auf einen bundespolitischen Einfluss hin.

Von 1978 bis 1990 stellte sich Ernst Albrecht als amtierender Ministerpräsident zur Wahl. Seine Popularität zeigt wie bei der Bundesregierung einen sinkenden Trend. Das Wahlergebnis der CDU ist jedoch zunächst 1986 besser als 1982. Dies scheint eher auf die schlechte Bewertung des SPD-Spitzenkandidaten, Karl Ravens, bei der Wahl 1982 im Vergleich zu Gerhard Schröder 1986 zurückzuführen sein. Ob die Zufriedenheit mit der damaligen Bundesregierung eine Rolle spielte, bleibt wegen der Nicht-Verfügbarkeit der Daten unklar. Der Grund für die negative Entwicklung der CDU-Ergebnisse von 1982 bis 1990 kann nicht allein in der Bewertung Albrechts gesucht werden, da er bei jeder Wahl zumin-

9 Zunächst folgte Gerhard Glogowski auf Gerhard Schröder im Amt des Ministerpräsidenten, als Letzterer nach Berlin wechselte. Glogowski musste jedoch nach einem Jahr wegen diverser Skandale zurücktreten, bevor er eine Landtagswahl bestreiten konnte. Sein Nachfolger Sigmar Gabriel kann deshalb als Nachfolger von Schröder als Spitzenkandidat angesehen werden.

10 Niedersächsische Wahlstudien der Forschungsgruppe Wahlen, die am Zentralarchiv für Empirische Sozialforschung (ZA) der Universität zu Köln zur Verfügung stehen: 1970 ZA-Nr. 0703, 1978 ZA-Nr. 2316, 1982 ZA-Nr. 1245, 1986 ZA-Nr. 1519, 1990 ZA-Nr. 1934, 1994 ZA-Nr. 2509, 1998 ZA-Nr. 3031, 2003 ZA-Nr. 867. Für die Wahl 1974 wurde keine Erhebung durchgeführt. 
dest besser als sein Herausforderer von der SPD beurteilt wurde. Dagegen könnte die bessere Bewertung des neuen Herausforderers Schröder gegenüber Ravens erklärt werden. Auch hier lässt sich wegen der fehlenden Datenbasis keine Aussage über den Bundesfaktor treffen.

\begin{tabular}{|c|c|c|c|}
\hline \multicolumn{4}{|c|}{$\begin{aligned} \text { Tabelle 1: Die Zufriedenheit mit der Bundesregierung und die Bewertung der Spitzenkandidaten } \\
\text { von CDU und SPD auf Landesebene in Niedersachsen, } 1970 \text { bis } 2003\end{aligned}$} \\
\hline \multirow[t]{2}{*}{ Landtagswahl } & \multirow[t]{2}{*}{$\begin{array}{c}\text { Zufriedenheit mit der } \\
\text { Bundesregierung }\end{array}$} & \multicolumn{2}{|c|}{$\begin{array}{l}\text { Durchschnittsbewertung der Spitzenkandidaten } \\
\text { bei der jeweiligen Landtagswahl }\end{array}$} \\
\hline & & $\mathrm{CDU}$ & SPD \\
\hline 1970 & $\begin{array}{c}\text { SPD-FDP: } \\
2,09 \\
(, 075)\end{array}$ & $\begin{array}{c}\text { Wilfried Hasselmann: } \\
1,84 \\
(, 088)\end{array}$ & $\begin{array}{c}\text { Alfred Kubel: } \\
1,89 \\
(, 090)\end{array}$ \\
\hline 1978 & $\begin{array}{l}\text { SPD-FDP: } \\
\text { nicht erhoben }\end{array}$ & $\begin{array}{c}\text { Ernst Albrecht: } \\
1,59 \\
(, 089)\end{array}$ & $\begin{array}{c}\text { Karl Ravens: } \\
1,04 \\
(, 088)\end{array}$ \\
\hline 1982 & $\begin{array}{l}\text { SPD-FDP: } \\
\text { nicht erhoben }\end{array}$ & $\begin{array}{c}\text { Ernst Albrecht: } \\
1,28 \\
(, 096)\end{array}$ & $\begin{array}{c}\text { Karl Ravens: } \\
0,34 \\
(, 059)\end{array}$ \\
\hline 1986 & $\begin{array}{l}\text { CDU-FDP: } \\
\text { nicht erhoben }\end{array}$ & $\begin{array}{c}\text { Ernst Albrecht: } \\
1,17 \\
(, 091)\end{array}$ & $\begin{array}{c}\text { Gerhard Schröder: } \\
0,44 \\
(, 081)\end{array}$ \\
\hline 1990 & $\begin{array}{c}\text { CDU-FDP: } \\
1,21 \\
(, 090) \\
\end{array}$ & $\begin{array}{c}\text { Ernst Albrecht } \\
0,92 \\
(, 095)\end{array}$ & $\begin{array}{c}\text { Gerhard Schröder: } \\
0,80 \\
(, 086)\end{array}$ \\
\hline 1994 & $\begin{array}{l}\text { CDU-FDP: } \\
-0,82 \\
(, 093)\end{array}$ & $\begin{array}{c}\text { Christian Wulf: } \\
1,33 \\
(, 083)\end{array}$ & $\begin{array}{c}\text { Gerhard Schröder: } \\
1,58 \\
(, 082)\end{array}$ \\
\hline 1998 & $\begin{array}{c}\text { CDU-FDP: } \\
-1,03 \\
(, 097)\end{array}$ & $\begin{array}{c}\text { Christian Wulff: } \\
1,07 \\
(, 088)\end{array}$ & $\begin{array}{c}\text { Gerhard Schröder: } \\
1,81 \\
(, 088)\end{array}$ \\
\hline 2003 & $\begin{array}{l}\text { SPD-Grüne: } \\
-0,87 \\
(, 095) \\
\end{array}$ & $\begin{array}{c}\text { Christian Wulff: } \\
1,54 \\
(, 082) \\
\end{array}$ & $\begin{array}{c}\text { Sigmar Gabriel: } \\
0,71 \\
(, 092) \\
\end{array}$ \\
\hline \multicolumn{4}{|c|}{$\begin{array}{l}\text { Mittelwerte einer von }-5 \text { bis }+5 \text { laufenden Skala (Zufriedenheit mit der Landesregierung: }-5 \text { sehr unzu- } \\
\text { frieden, }+5 \text { sehr zufrieden; Spitzenkandidaten: }-5 \text { sehr unsympathisch, }+5 \text { sehr sympathisch). } \\
\text { In Klammern sind die Standardfehler angegeben. } \\
\text { Die Werte der vor der jeweiligen Wahl amtierenden Ministerpräsidenten sind fett gedruckt. } \\
\text { Quelle: Niedersächsische Wahlstudien der Forschungsgruppe Wahlen im ZA Köln: } 1970 \text { ZA-Nr. 0703, } \\
\text { 1978 ZA-Nr. 2316, } 1982 \text { ZA-Nr. 1245, } 1986 \text { ZA-Nr. 1519, } 1990 \text { ZA-Nr. 1934, } 1994 \text { ZA-Nr. 2509, } \\
\text { 1998 ZA-Nr. 3031, } 2003 \text { ZA-Nr. 3867. }\end{array}$} \\
\hline
\end{tabular}

Ab der Landtagswahl 1990 stehen wieder Daten zur Zufriedenheit mit der Bundesregierung zur Verfügung. Diese ist höher als in den folgenden Wahljahren, jedoch niedriger als die Popularität der sozial-liberalen Koalition 1970. Auch wenn es schwer einzuschätzen ist, ob die Bundesregierung 1990 beliebt war oder nicht, könnte dieser Faktor den Sieg der SPD trotz der geringeren Popularität Schröders gegenüber Albrecht erklären. 1994 und 1998 erzielte die SPD mit ihrem inzwischen populären Ministerpräsidenten Schröder Wahlerfolge 
im Lande. Gleichzeitig sank jedoch die Zufriedenheit mit der damaligen schwarz-gelben Koalition im Bund. ${ }^{11}$ Auch dieser Faktor könnte zu den Wahlsiegen der SPD bei den Landtagswahlen 1994 und 1998 beigetragen haben.

Erstaunlich ist die sehr negative Einschätzung der SPD-Bundesregierung schon zu Beginn ihrer zweiten Amtszeit. Diese Bewertung könnte auch die Chancen der niedersächsischen SPD bei der Landtagswahl 2003 beeinträchtigt haben. Allerdings stand mit Sigmar Gabriel für die SPD auch ein Spitzenkandidat zur Wahl, dessen Popularität sich nicht mit der von Schröder in den vorangegangenen Landtagswahlen messen konnte. Daher ist noch unklar, ob die negative Einschätzung der Bundesregierung oder die geringe Popularität Gabriels im Vergleich zu seinem Gegner Wulff zur Niederlage der SPD führte.

Neben den Spitzenkandidaten zeigt Rita Süssmuth, die designierte Nachfolgerin Albrechts 1990, mit einem Wert von 2,35 eine sehr hohe Popularität. Dies dürfte mit ihrer engen Verbindung zur bundespolitischen Ebene und mit der dadurch entstandenen Präsenz in der Öffentlichkeit zusammenhängen (Süssmuth war 1990 sowohl Bundestagspräsidentin als auch Mitglied des CDU-Präsidiums). Dies lässt sich auch bei einigen Spitzenkandidaten erkennen: So war Ravens von 1974 bis 1978 Bundesminister für Raumordnung, Bauwesen und Städtebau in der von der SPD geführten Bundesregierung, womit der deutliche Popularitätsunterschied zwischen den Landtagswahlen 1978 und 1982 erklärt werden könnte. Auch Schröder war bei der Landtagswahl 1998 beliebter als bei den beiden vorangegangen Wahlen und dürfte von den Spekulationen um seine Kanzlerkandidatur profitiert haben. Landespolitiker, die einen deutlichen Bezug zur Bundesebene aufweisen, scheinen also beliebter zu sein als Politiker ohne diese Verbindung. Schlägt sich diese hohe Beliebtheit von Ravens und Schröder in einer hohen Wahlwahrscheinlichkeit für die SPD nieder, wäre dies als ein bundespolitischer Einfluss auf die Landtagswahl zu werten.

Der aufgezeigte Verlauf der Zufriedenheit mit der Bundesregierung und der Popularität von Spitzenkandidaten von CDU und SPD auf Landesebene konnte nur erste Hinweise auf bundes- und landespolitische Einflüsse auf Landtagswahlen geben. Insbesondere für die Wahlen, für die beide Indikatoren in die gleiche Richtung zeigen, muss anhand der Analyse individueller Wahlentscheidungen geklärt werden, ob einer der beiden Faktoren den Ausschlag gab.

\section{Zufriedenheit mit der Bundesregierung und Einschätzung der Spitzenkandidaten als Einflussfaktoren für die Wahlabsicht}

Die folgende Analyse muss auf fünf der acht Landtagswahlen beschränkt werden, da für die Wahlen 1978, 1982 und 1986 die Zufriedenheit mit der Bundesregierung nicht erhoben wurde. Die abhängige Variable in der logistischen Regressionsanalyse erfasst die Wahlabsicht für eine der Parteien der Bundesregierung mit dem Wert 1, alle anderen Wahloptionen dagegen mit dem Wert Null, Nichtwähler werden nicht berücksichtigt. Anhand dieser Modellierung soll geklärt werden, ob die Wahl einer an der Bundesregierung beteiligten Partei bei Landtagswahlen auf bundes- oder landespolitische Faktoren zurückzuführen ist.

11 Der Unterschied der Zufriedenheit zwischen 1994 und 1998 ist allerdings ausweislich eines statistischen Tests nicht signifikant. Dies könnte daran liegen, dass die Zufriedenheit der Wähler bereits bei der 1994 Wahl einen Tiefpunkt erreicht hatte. 
Dieses Vorgehen erspart die Anpassung der Erwartung über das Vorzeichen der Zufriedenheit mit der Bundesregierung an die jeweilige Regierung: Durch die Messung der Wahlabsicht anhand der Parteien der Bundesregierung sollte die Zufriedenheit immer ein positives Vorzeichen haben, da so auch die Wahrscheinlichkeit steigt, eine Partei der Bundesregierung zu wählen. Die Vorzeichen der Koeffizienten für die Spitzenkandidaten von CDU und SPD sind dagegen von der Regierungskoalition auf Bundesebene abhängig: Eine gute Bewertung eines Spitzenkandidaten der CDU sollte beispielsweise die Wahrscheinlichkeit, Parteien der Regierung Kohl zu wählen, erhöhen. Hier wird also ein positives Vorzeichen erwartet. Hingegen schwächt die gute Einschätzung eines CDU-Kandidaten die Wahrscheinlichkeit, 1970 und 2003 eine der Parteien der SPD-geführten Bundesregierung zu wählen. Hier ist also ein negatives Vorzeichen zu erwarten. Analog haben die Spitzenkandidaten der SPD einen positiven Einfluss auf die Wahl einer SPD-geführten Regierung, aber einen negativen Einfluss auf die Wahl einer CDU-geführten Regierung (vgl. Tabelle 2).

Die Zufriedenheit mit der Bundesregierung begünstigt wie erwartet bei allen fünf betrachteten Landtagswahlen die Wahl einer Partei der Bundesregierung. Auch die Vorzeichen der Koeffizienten für die Spitzenkandidaten gehen generell in die angenommene Richtung: Während der schwarz-gelben Koalition in Bonn, später Berlin, haben die Spitzenkandidaten der SPD einen negativen Effekt auf die Wahl einer Bundesregierungspartei, während der sozial-liberalen Koalition 1970 und der rot-grünen Regierungsmehrheit 2003 jedoch einen positiven. Dies gilt entsprechend auch für die Spitzenkandidaten der CDU. Lediglich bei Wulff kehrte sich 1994 dieser Zusammenhang um: Je besser man ihn bewertete, desto unwahrscheinlicher war es, dass man die CDU oder die FDP wählte, und umgekehrt. Die Ursache hierfür dürfte in Wulffs sehr positiver Bewertung liegen. Diese kam jedoch weniger durch die Anhänger der CDU als durch jene anderer Parteien zustande, was auch an der hohen Korrelation zwischen den Bewertungen Wulffs und Schröders $(0,534)$ deutlich wird. Die Bürger, die ihn positiv bewerteten, hielten gleichzeitig viel von Schröder und stimmten eher für die SPD. Seine Beliebtheit führte daher nicht dazu, dass sich eine Mehrheit der Wähler ihn als Ministerpräsidenten wünschte ${ }^{12}$ und die CDU vermehrt gewählt wurde. Das negative Vorzeichen bedeutet nicht, dass Wulff seiner Partei geschadet hat. Er hat ihr mit seiner sehr positiven Bewertung in Bezug auf Wählerstimmen von anderen Parteianhängern jedoch auch nichts genützt.

Hinsichtlich des relativen Einflusses bundes- und landespolitischer Faktoren ergibt sich somit ein gemischtes Bild. Die Zufriedenheit mit der Bundesregierung war bei den Wahlen 1990 und 1994 der stärkste Faktor. Insbesondere 1994 traten die Spitzenkandidaten im Lande in den Hintergrund. Aufgrund der sehr positiven Bewertung Schröders als Amtsinhaber wie auch des jungen und unverbrauchten Herausforderers Wulff schien eine Entscheidung auf Basis der Spitzenkandidaten schwierig zu sein, weshalb bundespolitische Erwägungen eine größere Rolle spielen konnten. 1990 hatte jedoch auch die Bewertung Schröders neben der Zufriedenheit mit der Bundesregierung großen Einfluss auf die Landtagswahl, während der immer noch besser als Schröder bewertete Albrecht es nicht vermochte, eine große Zahl an Stimmen für ihre Partei zu holen. 1998 und 2003 überwog der Einflussfaktor Kandidaten jenen der Zufriedenheit mit der Bundesregierung. Insbesondere der Effekt Wulffs ragt hierbei heraus. Für 1970 lässt sich hingegen kaum ein Unterschied

12 Vgl. Reinhold Roth, Die niedersächsische Landtagswahl vom 13. März 1994: Rot-grün honoriert und doch beendet, in: ZParl, 26. Jg. (1995), H. 2, S. 207. 


\begin{tabular}{|l|c|c|c|c|c|}
\hline Tabelle 2: Landes- und bundespolitische Einflüsse auf niedersächsische Landtagswahlen, 1970 und \\
1990 bis 2003
\end{tabular}

zwischen dem Einfluss der Zufriedenheit mit der Bundesregierung und dem des CDUSpitzenkandidaten Hasselmann erkennen. Beide sind wesentlich für die Wahlentscheidung, während von dem wieder kandidierenden SPD-Ministerpräsidenten Kubel keine Wirkung ausgeht. Der bundespolitische Einfluss könnte bei dieser Landtagswahl damit zusammenhängen, dass in Niedersachsen eine Große Koalition regierte und beide Spitzenkandidaten ähnlich positiv bewertet wurden. Wie auch bei der Wahl 1994 könnte dies zur stärkeren Beachtung der Bundespolitik bei der Wahlentscheidung geführt haben.

Wie oben gezeigt werden Spitzenkandidaten mit einer Verbindung zur Bundesebene unerwartet positiv bewertet. Haben diese Kandidaten ebenfalls eine außergewöhnliche Wirkung auf die Wahlentscheidung, könnte man dies als bundespolitischen Einfluss werten. Für Ravens, bei der Landtagswahl 1978 Bundesminister für Raumordnung, Bauwesen und Städtebau, können wir diese Vermutung aufgrund fehlender Daten nicht überprüfen. Das Ergebniss zum Einfluss der Popularität Schröders auf die Landtagswahl 1998 bestätigt diese Erwartung jedoch nicht. Dies macht ebenso wie der mangelnde positive Effekt Wulffs für seine Partei 1994 deutlich, dass große Beliebtheit eines Spitzenkandidaten nicht unbedingt zu mehr Stimmen für die jeweilige Partei führen muss. Daher scheint es unwahrscheinlich zu sein, dass die Bundespolitik in Form einer hohen Popularität eines Spitzenkandidaten, der über Verbindung zur bundespolitischen Ebene verfügt, die Landtagswahl beeinflusst.

Ob bundes- oder landespolitische Erwägungen bei einer Landtagswahl überwiegen, hängt wohl wesentlich von der wahlspezifischen Entscheidungssituation ab: Gibt es klare Alternativen im Lande, also beispielsweise Koalitionen nach politischen Lagern oder sehr starke Spitzenkandidaten, spielen landespolitische Einflüsse die Hauptrolle. Ist dies nicht der Fall, treten bundespolitische Einflüsse in den Vordergrund. 


\section{Zusammenfassung}

Zum einen stellt die Unklarheit bezüglich der Messung landes- und bundespolitischer Einflüsse ein generelles Problem der Individualdatenanalyse dar. Obwohl etliche Variablen in den umfangreichen Datensätzen zu Landtagswahlen vorhanden sind, ist eine Trennung der beiden Einflussfaktoren schwierig. Durch die Konsistenz der Bewertungsobjekte, also der Parteien, und der Verbindungen zwischen beiden Ebenen, die sich oft in Kandidaten manifestieren, ist es wahrscheinlich, dass auch die Wähler weder kognitiv noch evaluativ strikt zwischen beiden Ebenen trennen (können). Dies erschwert eine Messung des Einflusses von Bundespolitik auf Landtagswahlen. Zum anderen lässt sich voraussagen, dass hierdurch bei allen Landtagswahlen sowohl landes- als auch bundespolitische Einflüsse eine Rolle spielen. Es geht also weniger darum, die Existenz beider Einflussfaktoren zu beweisen, als vielmehr um die Voraussage ihres relativen Gewichts. Hierfür müssen Kontextbedingungen identifiziert werden, die den Einfluss der Faktoren stärken oder schwächen.

In Niedersachsen stellt die Landespolitik einen wichtigen Einflussfaktor dar. Wenn sich den Wählern auf der landespolitischen Ebene jedoch keine klaren Entscheidungskriterien anbieten, sei es durch Große Koalitionen oder durch ähnlich beliebte Spitzenkandidaten, erleichtert die Beachtung der bundespolitischen Ebene es, zu einer Entscheidung zu gelangen. Dieser Befund eines explorativen Vorgehens stellt zunächst ein nicht zu verallgemeinerndes Ergebnis dar, kann jedoch den Ausgangspunkt für die Identifizierung relevanter Kontextbedingungen und die Überprüfung ihrer Wirkung bei Landtagswahlen in allen 16 Bundesländern bilden.

\section{Hamburger Bürgerschaftswahlen 1978 bis 2004: Rückenwind oder Stimmungstief durch bundespolitische Einflüsse?}

\section{Roland Abold}

In regelmäßiger Folge erregen Hamburger Bürgerschaftswahlen eine Aufmerksamkeit, die weit über die Landesgrenzen des Stadtstaates hinausgeht. Dazu trägt nicht zuletzt die Tatsache bei, dass es mit vier vorgezogenen Neuwahlen seit Anfang der 1980er Jahre in keinem anderen Bundesland so häufig zu einem vorzeitigen Ende der Legislaturperiode gekommen ist wie in Hamburg. Die Wahl von 1991 wurde zudem in einem bisher einmaligen Vorgang nachträglich vom Landesverfassungsgericht für ungültig erklärt. 2001 kam erstmals in der Nachkriegsgeschichte mit der Schill-Partei eine Partei, die gerade gut ein Jahr existierte, aus dem Stand auf fast 20 Prozent der Stimmen. Ein weiterer politischer Paukenschlag war der Stimmengewinn der CDU bei der darauf folgenden Bürgerschaftswahl des Jahres 2004. ${ }^{1}$

1 Vgl. Patrick Horst, Die Neuwahl zur Hamburger Bürgerschaft vom 29. Februar 2004: die Hanseaten und Angela Merkel finden einen politischen Hoffnungsträger, in: ZParl, 35. Jg. (2004), H. 2, S. $252 \mathrm{ff}$. 